Review

\title{
Hybrid Mode Locked Fiber Ring Laser
}

\author{
Wenbo Li, Xiang Zhang \\ Department of Physics, University of Connecticut, Storrs CT 06269 USA
}

\begin{abstract}
Mode locked pulse generation has been reported using both active and passive mode locking schemes. Active mode locking technique has been proven to be an effective way to generate high-repetition-rate pulses by incorporating a modulator inside the laser cavity. Compared to actively mode locked lasers, passively mode locked lasers can generate pulse train at ultrashort pulse width but with relatively lower repetition rate. Thus, it is a brilliant idea to build a hybrid mode locked system combining both active and passive mode lockers. In this review, several hybrid mode locked fiber ring laser systems are discussed and summarized. Hybrid mode locking is a promising method to generate high speed ultrashort optical pulses for fiber-optic telecommunication system.
\end{abstract}

Keywords: fiber ring laser; hybrid mode locking; passive mode locking; rational harmonic mode locking; pulse compression

\section{Introduction}

The generation of stable high speed ultrashort pulses is very important for fiber-optic telecommunication system. Future transmission systems may operate at data rate $250 \mathrm{~Gb} / \mathrm{s}$ or more [1]. Among various platforms, active harmonic mode-locking has proven to be an effective way to generate high-repetition-rate pulses by incorporating an electro-optical intensity modulator, such as $\mathrm{LiNiO} 3$ modulators or electric absorption modulators (EAM) inside the laser cavity. In particular, the implementation of rational harmonic mode-locking technique is able to overcome the modulator bandwidth limitation and further increase the repetition rate [2]. However, with rational harmonic mode-locking, the temporal pulse duration is usually limited to several picoseconds. Furthermore, the amplitudes of pulse train suffer from severe fluctuations in the time domain. This amplitude unevenness is not good for fiber optic communication systems. Several researchers have carried out experiments to either compress the optical temporal width or suppress the supermode noise to improve the laser stability. These schemes include (1) adiabatic soliton compression techniques [3-4], (2) multiple-step compression based on self phase modulation in highly nonlinear fibers or semiconductor optical amplifiers (SOA) [5-6], (3) a comb-like profiled fiber (CPF) to emulate dispersion decreasing fiber [7-8], (4) saturable optical absorbers such as nonlinear optical loop mirror (NOLM) [9-11].

Compared to actively mode-locked lasers, passively mode-locked fiber lasers have the advantage of generating pulse train at ultrashort pulse width. Recently, compact passively mode-locked fiber lasers have been successfully constructed to generate subpicosecond pulses by using a saturable absorber (SA) such as semiconductor saturable absorber mirror (SSAM) [12], graphene [13], nonlinear polarization rotation (NPR) technique [14, 15] and nonlinear fiber loop mirror [16]. Despite the fact that those passively mode locked lasers can produce ultrashort pulses, the passive mode-locking scheme suffers from the drawback of 
low repetition rate, which is generally at MHz-level. A possible solution would be to build a hybrid mode-locked scheme to combine this two mode-locking methods.

In this review, three types of hybrid mode locked schemes [17-19] were discussed and summarized both numerically and experimentally. All the schemes incorporate saturable absorbers into the rational harmonic mode locked laser cavity, which were able to generate optical pulse trains with high repetition rate and short pulse width.

\section{Hybrid mode locked fiber ring laser using charcoal nano-particles}

Since the first demonstration of graphene saturable absorber for passively mode-locked erbium-doped fiber lasers (EDFLs), versatile graphene samples in different forms have been developed, including single-layer graphene [20], few-layer graphene [21], multi-layer graphene [22], graphene polymer [23], graphene composite [24], graphene solution [25] and graphite nano-particle [26] etc., which progressively show the capabilities on initiating the ultrafast saturable absorption in the EDFL cavity. Recently, Singh et al demonstrated a green and simple method to synthesis the graphene nano-sheets from a pencil using the electrochemical exfoliation [27]. The bulk charcoal structure in a pencil is confirmed to be similar with graphite that contains multi-layer graphene. In the meantime, Lin's group also obtained the charcoal nano-particles by simply polishing the pencil [28], and presented that even the unprocessed charcoal nano-particles possess the ability of saturable absorption.

\subsection{Experimental Setup and Working Principle}

The experiment setup of fiber ring laser system is shown in Figure 1. The system consists of a $23 \mathrm{~m}$ long Er-doped fiber, a WDM coupler, a 980nm laser diode, a 10\% output coupler, an isolator, a polarization controller, a Mach-Zehnder type modulator and a synthesizer. All components were connected by regular single-mode fiber. We use a 980nm laser diode to pump the EDF and a 980/1550 wavelength division multiplexer (WDM) is involved to deliver the pumping power. The isolator is placed in the ring system to decide circulation direction and avoid feedback. The PC is inserted in the system to control the intra-cavity polarization. The synthesizer is used to generate the RF signal to drive the Mach-Zehnder type modulator which has a bandwidth of $11 \mathrm{GHz}$. The charcoal nano-particles are confined between two SMF patchcord connectors side the ring cavity. The output optical coupler provides $90 \%$ feedback ratio and $10 \%$ output coupling ratio. The triturated charcoal nanoparticles which are mechanically polished from the pencil were directly brushed onto the end-face of single-mode fiber (SMF) patchcord connector to be the saturable absorber for the fiber ring laser system using the imprinting-exfoliation-wiping method [26].

A $20-\mathrm{GHz}$ pulse train can be obtained from the ring cavity shown in Figure 1 with or without the charcoal nano-particles through rational harmonic mode locking (RHML). The fiber laser with a MZ modulator incorporated is actively mode-locked by carefully tuning the frequency of the RF signal and the polarization controller. When the fundamental frequency of the cavity is $f c$ and the modulation frequency fm satisfies the condition $f m=(n+1 / p) f c$, where $\mathrm{n}$ and $\mathrm{p}$ are both integers, the laser resonator operates in the rational harmonic modelocking (RHML) regime and pulses with a repetition rate of $(\mathrm{np}+1) \mathrm{fc}$ can be produced. In our case, $\mathrm{fc}$ is $2.8 \mathrm{MHz}$, and $\mathrm{fm}$ is set at $(3571+1 / 2) \mathrm{fc} \approx 10 \mathrm{GHz}$ to realize 2 nd order RHML. 


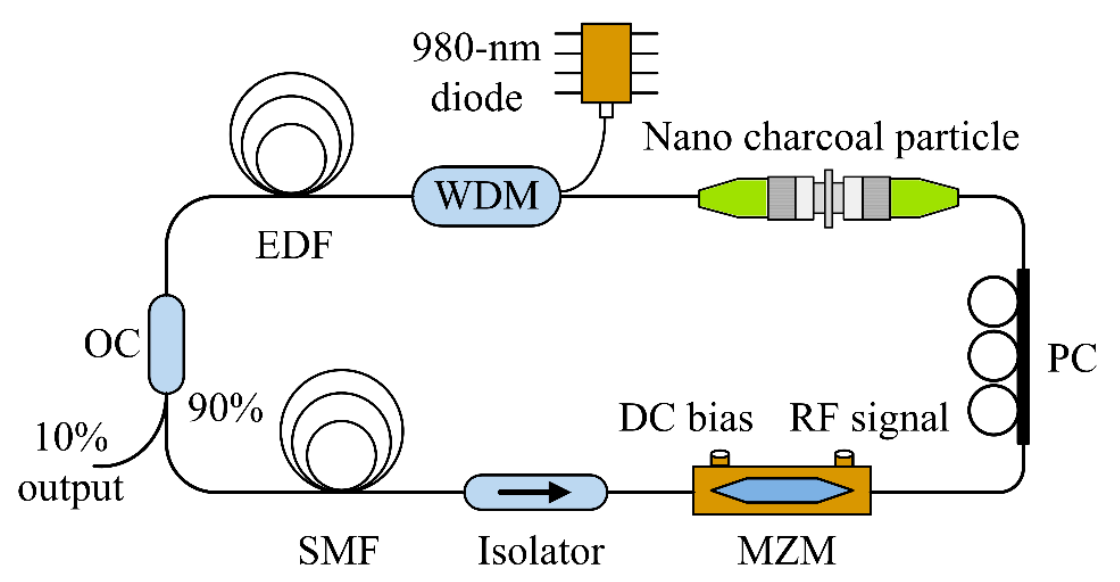

Figure 1: The schematic diagram of the fiber ring laser system with charcoal nano-particles inside the cavity. WDM: wavelength-division multiplexer, PC: polariztion controller.

The saturable absorbance of charcoal nano-particle is characterized by fiber ring laser illumination. The charcoal nano-particles used in the absorption measurement is the same sample as used in the laser experiment. The optical absorption of charcoal nano-particle is reduced under high optical power illumination because the carrier transition from valence band to conduction band is forbidden by the Pauli blocking effect [29]. The optical absorbance $\alpha$ of charcoal nano-particle is correlated with the linear absorbance (qlin), the nonlinear absorbance ( $\left.\mathrm{q}_{\text {non }}\right)$, as described by [17]:

$$
\alpha=q_{\text {lin }}+\frac{q_{\text {non }}}{1+I_{\text {in }} / \mathrm{I}_{\text {sat }}} \approx q_{\text {lin }}+q_{\text {non }}-\frac{q_{\text {non }}}{I_{\text {sat }}} I_{\text {in }}
$$

where $\mathrm{I}_{\text {in }}$ denotes the input intensity, $\mathrm{I}_{\text {sat }}$ the saturation intensity of charcoal nano-particle. The saturable absorption of charcoal nano-particles is shown in Figure 2(a) with a modulation depth of $36 \%$. Fitting this curve obtains the linear loss of qlin $=0.37$, the nonlinear loss of $\mathrm{q}_{\text {non }}=0.48$, and the saturation intensity of $\mathrm{I}_{\mathrm{sat}}=103 \mathrm{~kW} / \mathrm{cm}^{2}$ which is a relatively low intensity for initiating the saturable absorption. The saturable transmittance is also shown in Figure 2(b), with transmittance from 0.21 to 0.58 when increasing the input intensity from $16 \mathrm{~kW} / \mathrm{cm}^{2}$ to $1129 \mathrm{~kW} / \mathrm{cm}^{2}$.
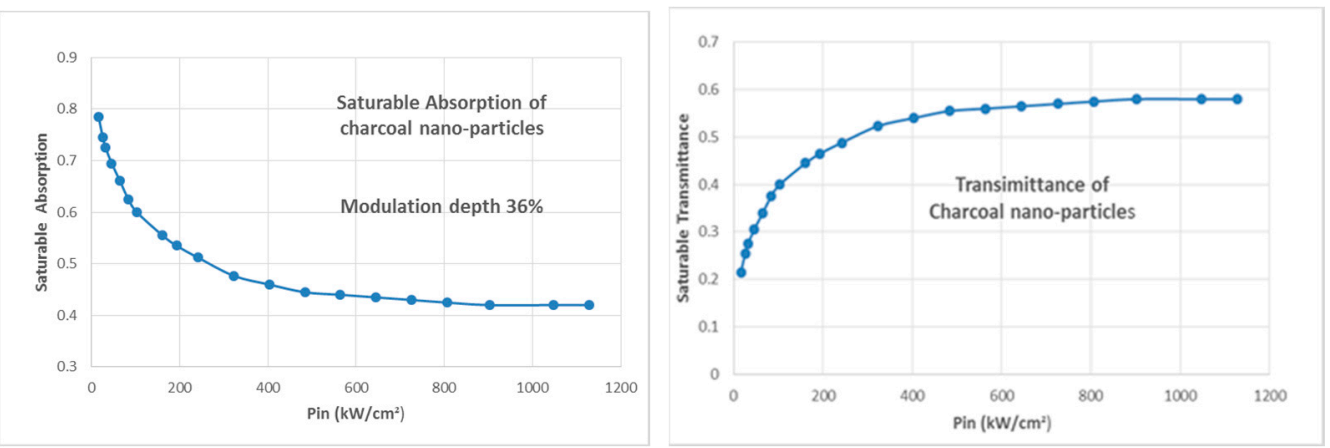

Figure 2: Saturable absorption (a) and Transmittance (b) of charcoal nano-particle

\subsection{Numerical simulation method}

The mode locked pulse evolution of the fiber ring laser cavity in Figure 1 can be simulated by solving equations from 3 to 6 . Equation 3 is an extended nonlinear Schrödinger equation $[30,31]$ which was used to model the gain medium, EDF in Figure 1, of the fiber ring laser. In equation $3, A(z, T)$ is the slowing varying amplitude of the pulse envelope, $\beta_{2}$ and $\beta_{3}$ are 
the second and third order dispersion parameters, respectively. $\gamma$ represents $\chi^{3}$ nonlinearity parameter, and $\Omega_{\mathrm{g}}$ is the gain bandwidth of the EDF. $\mathrm{g}$ is the gain of the EDF, which can be modeled by

$$
g=g_{0} /\left(1+E / E_{\text {sat-gain }}\right)
$$

where $\mathrm{g}_{0}$ is the small signal gain, $\mathrm{E}$ is the energy of the pulse envelop. Esat-gain represents the gain saturation energy of the EDF. Split-step Fourier method $[32,33]$ was used to solve the equation (3) to simulate the pulse transmission in EDF. The pulse evolution in single mode fiber (SMF) which composes the ring laser cavity can also be simulated by solving equation 3 if we set the gain parameter $g$ as 0 . The saturable absorption property of the charcoal nanoparticles incorporated into the cavity was modeled by equation 4 . The $90 / 10$ coupler in our fiber ring laser system as in Figure 1 can be simulated by solving equation 5 where R equals to $90 \%$. The Lithium Niobate modulator can be simulated by solving equation 6 , where $V_{\pi}$ is the required voltage for a $\pi$ phase shift between two arms of the modulator, $V(t)$ is the voltage applied to the modulator. In this scheme, the modulator is driven by a RF signal at frequency $\mathrm{f}_{\mathrm{m}}$. Thus, $V(t)=V_{b}+V_{m} \sin \left(\omega_{m} t\right)$ in which $\mathrm{V}_{\mathrm{b}}$ is the DC voltage bias of the modulator, $\mathrm{V}_{\mathrm{m}}$ is the amplitude of the RF signal. The numerical simulation was initiated by launching into the system a seed pulse with small amplitude. The pulse evolution within the ring cavity is then iteratively modeled until a steady state is reached after many roundtrips.

$$
\begin{gathered}
\frac{\partial A(z, T)}{\partial z}+\frac{i}{2} \beta_{2} \frac{\partial^{2} A(z, T)}{\partial T^{2}}-\frac{1}{6} \beta_{3} \frac{\partial^{2} A(z, T)}{\partial T^{2}}= \\
\frac{g}{2} A(z, T)+\frac{g}{2} \frac{1}{\Omega_{g}^{2}} \frac{\partial^{2} A(z, T)}{\partial T^{2}}+i \gamma|A(z, T)|^{2} A(z, T) \\
A(z . T)_{\text {out }}=A_{\text {in }}(z, T) \cdot T=A_{\text {in }}(z, T)\left(1-q_{\text {lin }}-\frac{q_{\text {non }}}{1+\frac{|A(z, T)|^{2}}{P_{\text {sat }}}}\right) \\
A(z, T)_{\text {out }}=R \bullet A_{\text {in }}(z, T) \\
A(z, T)_{\text {out }}=\cos \left(\frac{\phi(t)}{2}\right) A_{\text {in }}(z, \mathrm{~T}) \quad \text { with } \quad \phi(t)=\pi \frac{V(t)}{V_{\pi}}
\end{gathered}
$$

It should be mentioned that the asymptotic state is independent on the seed pulse. Pulses having the same characteristics can be obtained as well when pumped with white noise in the simulation.

\subsection{Experimental results and discussion}

Figure 3 shows the RF spectrum of the output pulse from the rational harmonic mode locking fiber ring laser with (b) or without (a) charcoal nano-particles in the cavity. Many side-bands are observed in Figure 6(a). The highest peak corresponds to the frequency of the fiber ring laser which is $20 \mathrm{GHz}$. The separation between these sidebands is equal to the fundamental cavity frequency of the fiber ring laser $\mathrm{f}_{\mathrm{c}}=2.8 \mathrm{MHz}$ so they are assumed to be the supermodes of the fiber ring laser. These supermodes cannot be removed by tuning the modulation frequency $\mathrm{f}_{\mathrm{m}}$ and polarization controller in the cavity. However, no supermodes 
were observed in Figure 6(b) with the charcoal nano-particles inside the ring cavity. The reduction of the supermodes in the RF spectrum can improve the stability of optical pulse generation in rational harmonic mode locking. The reduced amplitude of the supermodes in the fiber ring laser is the result of the saturable absorption character of the charcoal nanoparticles.

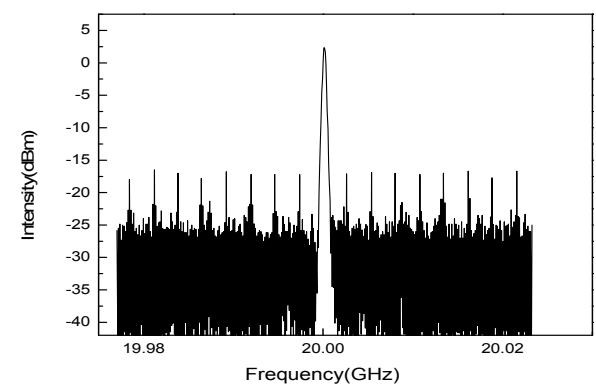

(a)

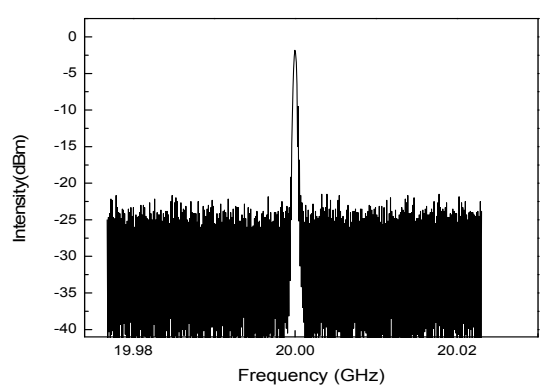

(b)

Figure 3: RF spectrum of rational harmonic mode locking at $20 \mathrm{~Gb} / \mathrm{s}$. (a) without the charcoal nanoparticles in the fiber ring laser cavity and (b) with the charcoal nano-particles in the fiber ring laser cavity.

Figure 4(b) shows the experimental fiber ring laser pulse shape, autocorrelation pulse with pulse width $\sim 5.6 \mathrm{ps}$, without charcoal nano-particles inside the ring. This result is in good agreement with the numerical simulation result, which is shown in Figure 4(a). The charcoal nano-particles inside the cavity can greatly improve the pulse shortening mechanism of the fiber ring laser due to their saturable absorption character. Figure 4(d) shows the experimental autocorrelation pulse with charcoal nano-particles inside the ring with pulse width $\sim 3.2 \mathrm{ps}$, about $57 \%$ of the pulse width without charcoal nano-particles. This experimental result is also in good agreement with the numerical simulation result, which is shown in Figure 4(c). Thus, with charcoal nano-particles inside the ring, shorter pulses can be generated with improved stability. The optical spectrum without charcoal nano-particles in the ring centered at $1561 \mathrm{~nm}$, with a $3-\mathrm{dB}$ bandwidth of $0.80 \mathrm{~nm}$, as shown in Figure 5(a). With charcoal nanoparticles, the bandwidth of the optical spectrum is broadened to $1.4 \mathrm{~nm}$, as shown in Figure $5(b)$.
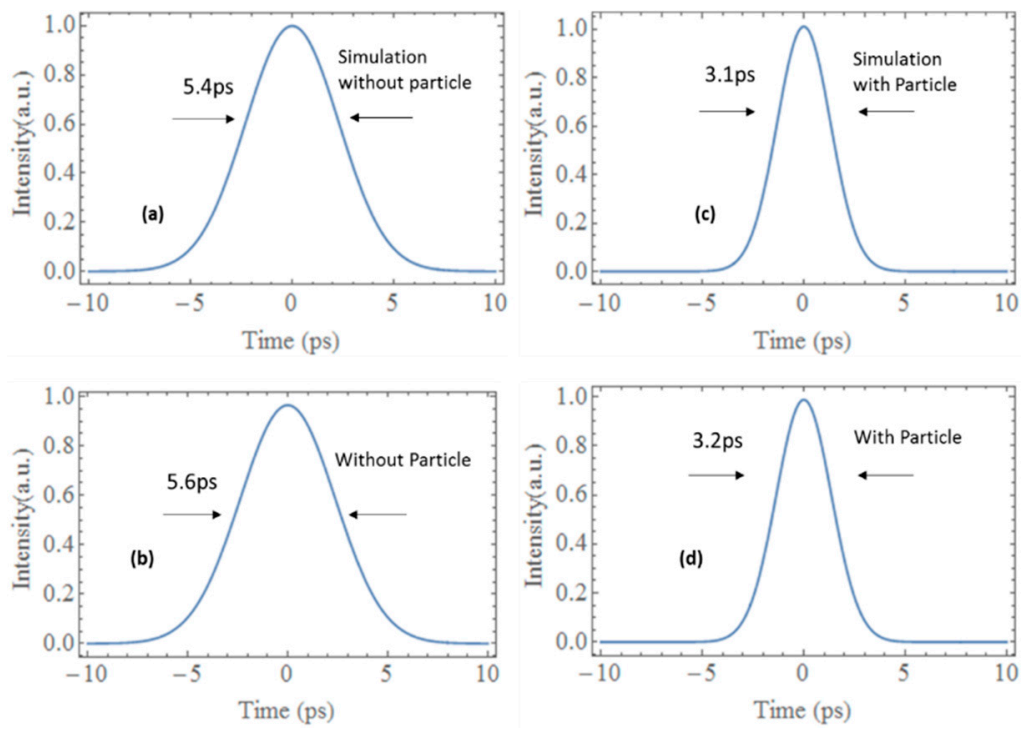

Figure 4: Pulse shape of the fiber ring laser. (a) Simulated pulse shape without nano-particles in the ring, 
pulse width $\sim 5.4$ ps. (b) Autocorrelation pulse without nano-particles inside the ring, pulse width $\sim 5.6 \mathrm{ps}$. (c) Simulated pulse shape with nano-particles in the ring, pulse width $\sim 3.1 \mathrm{ps}$. (d) Autocorrelation pulse with nano-particles inside the ring, pulse width $\sim 3.2 \mathrm{ps}$.

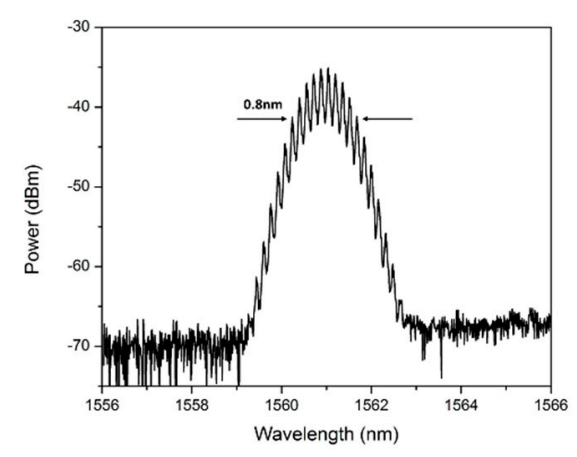

(a)

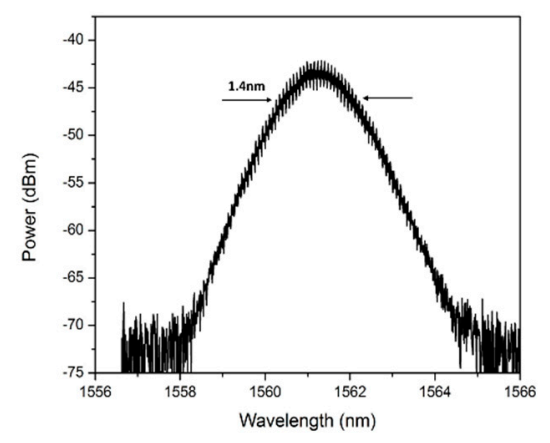

(b)

Figure 5: Optical Spectrum of output pulse (a) without charcoal nano-particle (b) with charcoal nanoparticle

\section{Hybrid mode locked fiber ring laser using graphene saturable absorber}

Recent advances in materials have further expanded opportunities for robust and costeffective pulse lasers [34-36]. Since the initial report by Bao et al. [37], mode-locked fiber lasers based on graphene saturable absorbers have attracted much attention due to their outstanding properties including ultrafast recovery time, wide range wavelength tunability, low saturation power, large modulation depth, and high damage threshold [38-40]. Thus, graphene based saturable absorber is a promising candidate to realize hybrid mode locking.

\subsection{Experimental Setup and Working Principle}

An experimental setup similar to Figure 1 can be used to realize hybrid mode locking as shown in Figure 6 with Graphene SA replacing nano-charcoal inside the fiber ring cavity. The length of the SMFs is carefully adjusted to ensure the insertion of the SA does not drift the cavity's fundamental frequency significantly.

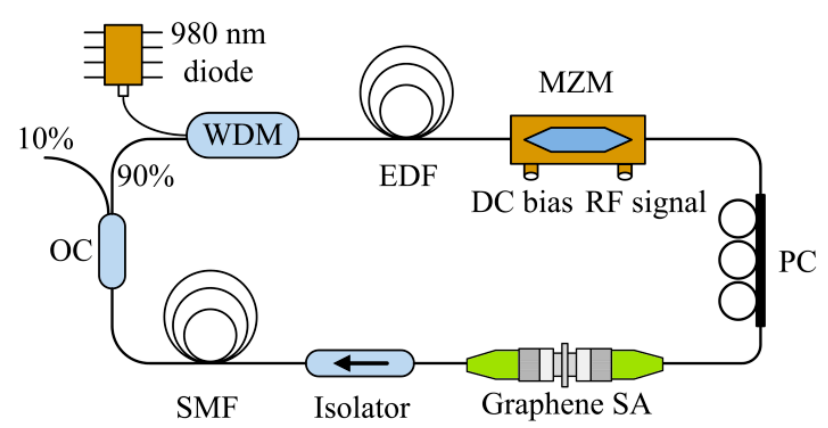

Figure 6. Schematic of the hybrid mode-locked fiber ring laser. EDF: Er-doped fiber, MZM: MachZehnder modulaor, PC: polarization controller, SA: saturable absorber, SMF: single-mode fiber, OC: optical coupler, WDM: wavelength-division multiplexer

To characterize the prepared graphene saturable absorber, the intensity dependent absorption was measured as illustrated in Figure 7. The optical transmittance of the graphene absorber is correlated with the linear absorption $\alpha_{\text {lin }}$ and nonlinear absorption $\alpha_{\text {non }}(\mathrm{I})$ 
expressed as:

$$
T(I)=\exp \left[-\left(\alpha_{\text {non }}(I)+\alpha_{\text {lin }}\right)\right]
$$

The saturable absorbance $\alpha_{\text {non }}(I)$ is given by:

$$
\alpha_{\text {non }}(I)=\alpha_{0}\left(1+\frac{I}{I_{\text {sat }}}\right)^{-1}
$$

where $I_{\text {sat }}$ is the saturation intensity.

(a)

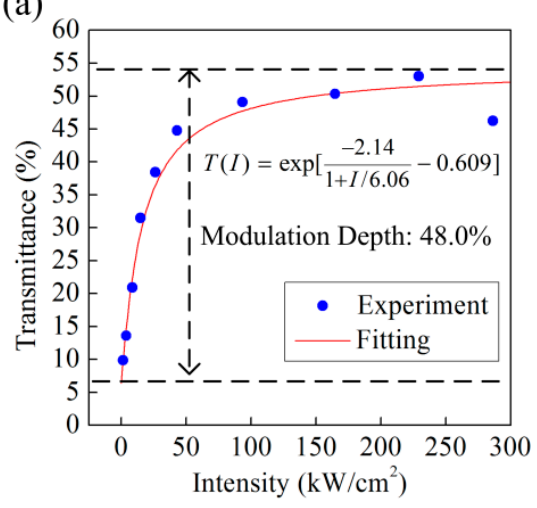

(b)

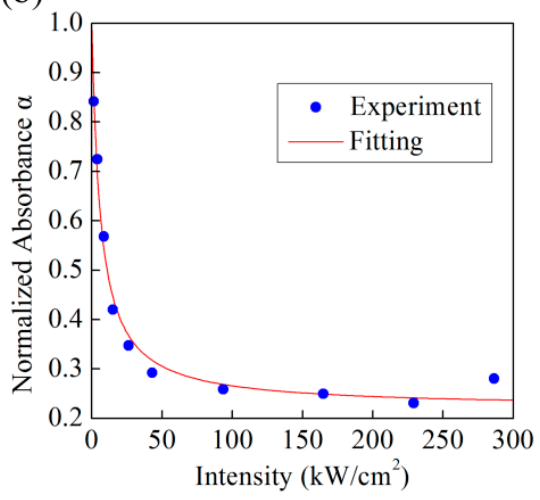

Figure 7. (a) Transmittance and (b) normalized absorption of the graphene SA as a function of input intensity

\subsection{Numerical simulation result}

The numerical simulation of the laser operation can be conducted [19]. Light propagation inside the laser cavity can be simulated by solving equation (3) to (6) by using the split-step Fourier method [41-43]. Simulation was initiated by launching into the system a seed pulse with small amplitude. The pulse evolution within the ring cavity is then iteratively modeled until a steady state is reached after many roundtrips.

Figure 8 and Figure 9 depict the simulated pulse development and the steady-state solutions of the output pulses, respectively. The FWHM is 5.35 ps when operated solely in the RHML (rational harmonic mode locking) regime, and narrowed by a factor of 0.55 to $2.95 \mathrm{ps}$ in the hybrid mode-locking regime. It should be mentioned that the asymptotic state is independent on the seed pulse. Pulses having the same characteristics can be obtained as well when pumped with white noise in the simulation.

(a)

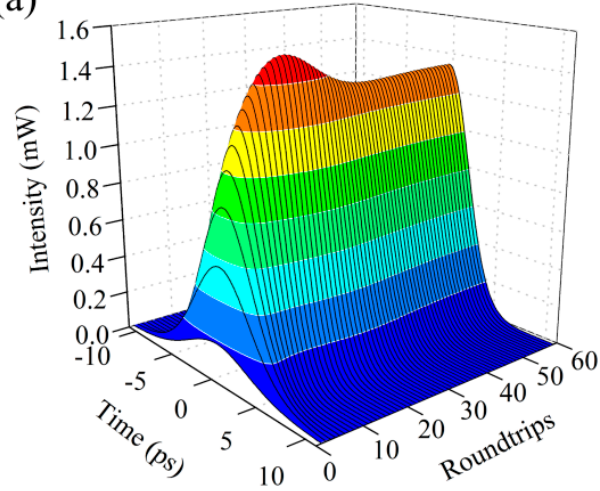

(b)

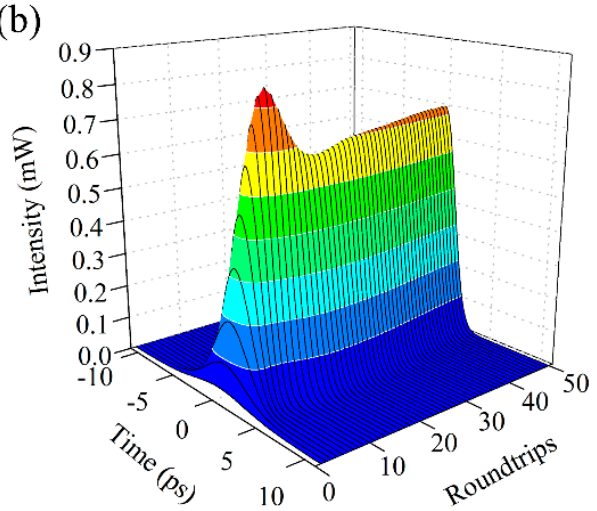

Figure 8. Simulated pulse generation from fiber lasers implementing (a) only rational harmonic active mode-locking and (b) hybrid mode-locking. 


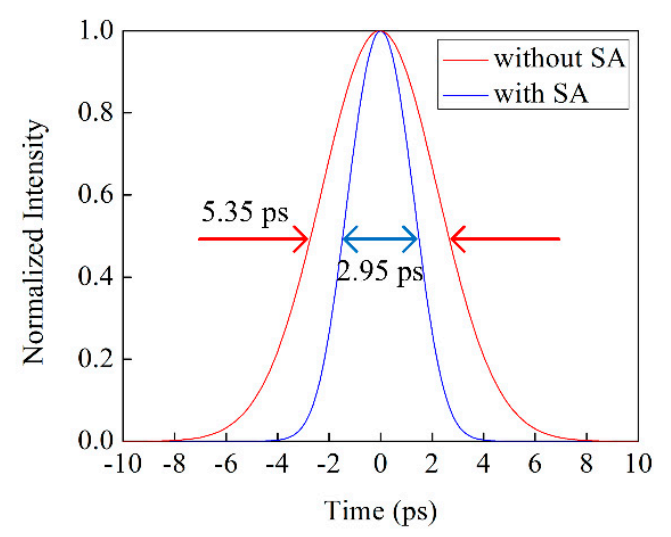

Figure 9. Comparison of the simulated output pulses generated from fiber lasers implementing rational harmonic active mode-locking and hybrid mode-locking.

\subsection{Experimental results and discussion}

The combination of an active and passive mode locker results in the improvement of the laser performance by compressing the pulse duration, as can be seen in Figure 10. The FWHM of the pulse is shortened to $2.82 \mathrm{ps}$, indicating a compression factor of 0.53 . Meanwhile, it is worth noting that the bandwidth of the optical spectrum is broadened to $1.38 \mathrm{~nm}$, leading to a TBP value of 0.479 . Due to the insertion loss introduced by the SA, the output power is reduced to $-2.8 \mathrm{dBm}(0.52 \mathrm{~mW})$ and the signal-to-noise ratio is $15 \mathrm{~dB}$.

(a)
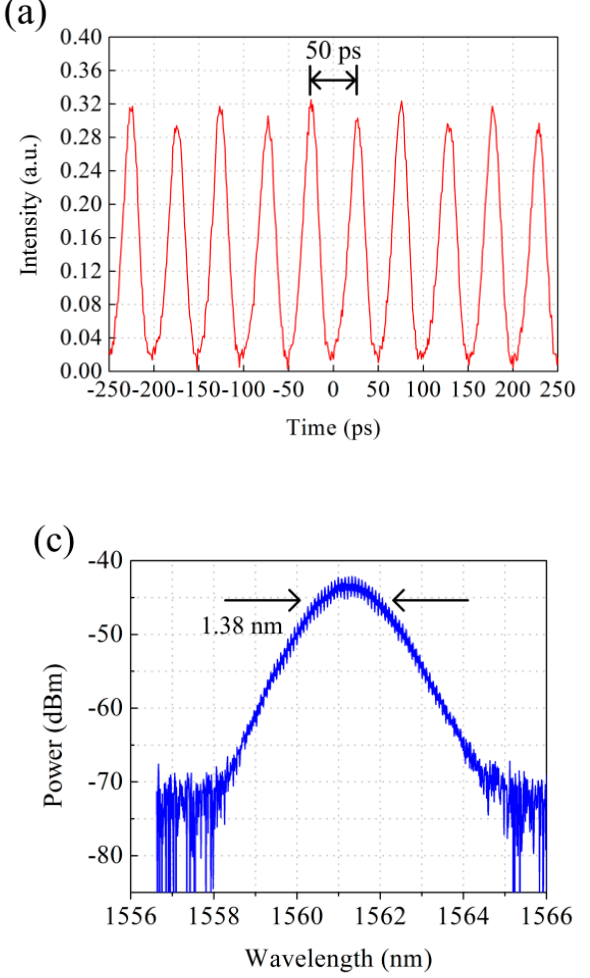
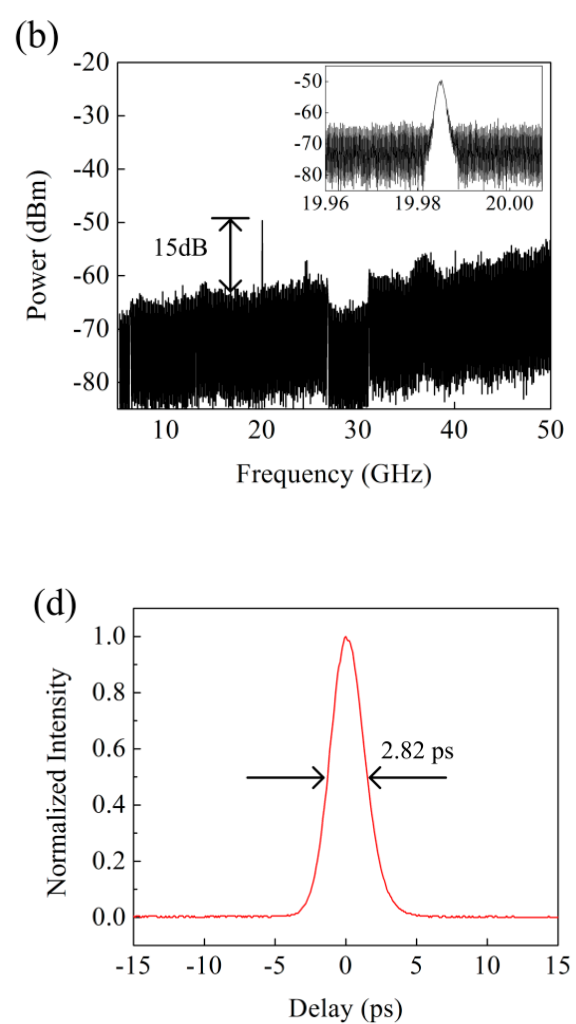

Figure 10. The measured (a) oscilloscope trace, (b) RF spectrum, (c) optical spectrum and (d) autocorrelation trace of the $20-\mathrm{GHz}$ pulse train generated by the hybrid mode-locked laser using graphene saturable absorber. 
In this hybrid mode locking scheme, the role of the active mode locker is to produce a high-speed optical pulse train. The modulator is capable of being driven at a frequency equal to a high multiple of relatively low fundamental frequency, thus the generated pulse train has a high repetition rate at the level of a few GHz. Furthermore, by applying the RHML technique, even higher repetition rate can be easily reached. However, this is rarely accomplished by passive mode lockers, especially graphene saturable absorbers. On the other hand, the passive graphene mode locker acts as a pulse compressor. This is expected since the recovery time of graphene is much shorter than the pulse width, hence the transmittance reacts to the instantaneous intensity of the injected light. As can be seen in Equation (8), the central part of the pulse with higher intensity experiences less absorption than the leading and trailing edges, which gives rise to pulse narrowing.

\section{Hybrid mode locked fiber ring laser based on NPR in PCF}

The nonlinear polarization rotation (NPR) technique combined with a polarizer can induce an intensity depended loss in the cavity, and has been used to achieve ultrashort pulses in fiber lasers. In [44], Luo et al constructed a L-band passively mode-locked fiber laser utilizing the NPR technique and generated pulses with full width at half maximum (FWHM) 458.7 fs. However, the pulse repetition rate is only at $8.6 \mathrm{MHz}$. Liu et al reported the generation of a stable passive $23^{\text {rd }}$ harmonic mode-locked pulse train at $230 \mathrm{MHz}$ with a pulse width of 0.44 ps [45]. Despite the fact that those NPR based passively mode locked fiber lasers can produce ultrashort femtosecond pulses, they suffer from the drawback of low repetition rate (only at $\mathrm{MHz}$ level) with respect to the total cavity length, which limits their applications in high speed fiber optic communications.

\subsection{Experimental Setup and Working Principle}

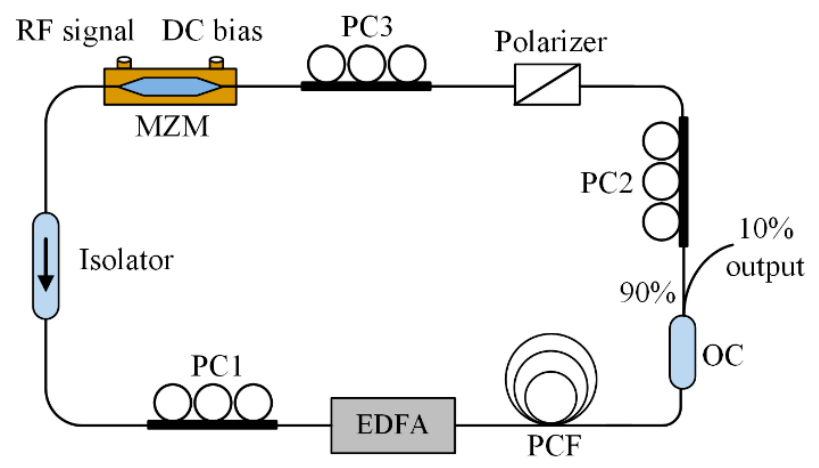

Figure11. Experiment setup of the hybrid mode-locked fiber ring laser based on the combination of rational harmonic mode locking and the passive nonlinear polarization rotation technique. EDFA: Er-doped fiber amplifier, MZM: Mach-Zehnder modulator, PC: polarization controller, OC: optical coupler.

Figure 11 shows the experimental setup of the proposed hybrid mode-locked fiber ring laser system. The gain of the fiber laser is provided by an EDFA, which consists of a 23-m long Erbium-doped fiber (EDF) and a 980-nm pump laser diode. $\mathrm{A} \mathrm{LiNbO}_{3} \mathrm{Mach}-Z \mathrm{Zehnder}$ modulator (MZM) driven by $\sim 10 \mathrm{GHz}$ radio frequency (RF) signal is utilized for rational harmonic mode-locking. Because the loss of the $\mathrm{LiNbO}_{3}$ modulator is polarization sensitive, a polarization controller PC3 is inserted at the input port of the modulator. An optical isolator in the cavity is to ensure unidirectional propagation of the laser mode. A highly nonlinear 
photonic crystal fiber (PCF), a inline polarizer and two PCs (PC1 and PC2) are used to generate nonlinear polarization rotation effect. If we remove polarization controllers $\mathrm{PC} 1$, PC2 and the polarizer, then there is no NPR effect in the cavity. The polarization controllers used here utilizes stress-induced birefringence of the fiber to create independent wave plates to alter the polarization of the transmitted light. The fractional wave plate is created by winding a short length of single mode fiber around a spool. Typically, the insertion loss is very low $(\sim 0.2 \mathrm{~dB})$. Compared to polarization controllers fabricated using bulk components, which use the $\lambda / 4$ or $\lambda / 2$ wave plates based on the birefringence of a crystal, fiber polarization controllers (stress induced) do not require fiber-to-free-space coupling; thus they are very easy to incorporate in a fiber cavity with low loss [46]. The laser output is coupled out using a 90:10 coupler. All the components are connected by standard single-mode fibers (SSMFs).

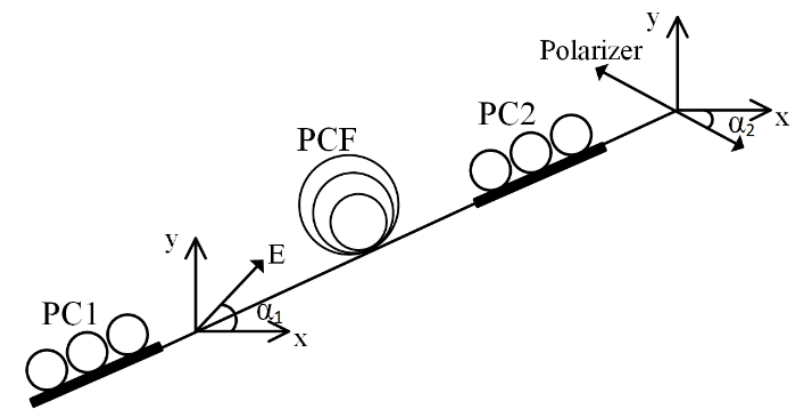

Figure 12. Operation principle of NPR. E: electric field, $\mathrm{x}$ : fast axis of PCF, y: slow axis of PCF, PC: polarization controller.

The PC-PCF-PC-polarizer structure can introduce the intensity-dependent loss and the transmission principle is shown in Figure 12. In Fig.2, $\alpha_{1}$ is the angle between the fast axis of the PCF and the polarization direction of the input signal before entering the PCF. E is the electric vector of input signal. $\alpha_{2}$ is the angle between the fast axis of PCF and the polarization direction of the in line polarizer. The Kerr nonlinearity of the PCF can generate a rotation of polarization state, which depends on the pulse intensity. The transmission introduced by NPR can be expressed as [47]:

$$
\begin{gathered}
\mathrm{T}=\cos ^{2} \alpha_{1} \cos ^{2} \alpha_{2}+\sin ^{2} \alpha_{1} \sin ^{2} \alpha_{2}+\frac{1}{2} \sin 2 \alpha_{1} \sin 2 \alpha_{2} \cos \left(\Delta \varphi_{L}+\Delta \varphi_{N L}\right) \\
\Delta \varphi_{L}=\left(\mathrm{n}_{x}-\mathrm{n}_{y}\right) / \beta \mathrm{L} \\
\Delta \varphi_{N L}=-\left(\frac{1}{3}\right) \gamma \mathrm{PL} \cos \alpha_{2}
\end{gathered}
$$

where $\beta=2 \pi / \mathrm{L}$ is the propagation constant; $\Delta \varphi_{\mathrm{L}}, \Delta \varphi_{\mathrm{NL}}$ are the linear and nonlinear phase changes; and $\mathrm{L}, \mathrm{n}_{\mathrm{x}}, \mathrm{n}_{\mathrm{y}}, \gamma$ are the length, linear birefringence coefficient of fast axis and slow axis and nonlinear coefficient of the PCF. $\mathrm{P}$ is the instantaneous power of input signal. The quantities $\alpha_{1}$ and $\alpha_{2}$ which determine the transmission through the NPR structure can be adjusted by changing the two polarization controllers PC1 and PC2. 


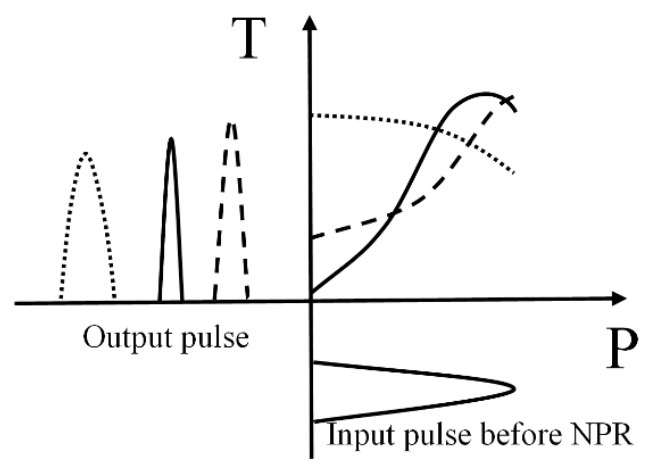

Figure 13. Conceptual illustration of effects of different transmission curves induced by NPR technique on pulse shaping.

As shown in equation 9, a cosine curve relation between the transmittivity and the instantaneous power can be plotted. The value of $\alpha_{1}$ and $\alpha_{2}$ will not only decide the offset and the amplitude of the cosine curve, but also affect the period of the cosine curve. Tuning PC1 and PC2 properly to change the valuse of $\alpha_{1}$ and $\alpha_{2}$, then the transmission curve can be varied as shown in Equation 9. When the rational harmonic mode-locked $30 \mathrm{GHz}$ pulses go through the NPR mechanism, they experience pulse shaping. Therefore, different transmittive curves will result in different pulse shaping mechanisms as suggested by Figure 13. The three transmission curves in Figure 13 correspond to three differetn $\alpha_{1}$ and $\alpha_{2}$ sets. If the NPR induced transmittive curve is the dotted line as shown in Figure 13, the low intensity part of the pulse (pulse wings) will have high transmission while the high intensity part (pulse center) will experience low transmission. In this case, the NPR somehow acts as a pulse equalizer which can reduce the intensity fluctuations of the pulse train; however due to the high transmission of pulse wings, it will also broaden the pulse width meanwhile. The dashed line in Figure 13 corresponds to a state that the high intensity part (pulse center) of the input pulse experiences little loss, which the low intensity parts (pulse wings) undergo high loss. The NPR in this state has the same funtionality as a satruable absorber, thus only leading to pulse compression. Compared to a saturable absorber, the NPR technique has more flexibility in acquiring various transmittive curves. When the tranmission curve is adjusted to the solid line shape, where the pulse wings still undergo great loss, the pulse center will experience a bit higher loss than its adjacent part. Therefore, at this sate, the pulse wdith is narrowed due to the high absorbtion of the leading and trailing edges and if the pulse peak intensity suddenly rises of falls due to the environmental distrubance or mode competition, the transmission loss of the NPR will also rise or fall correspondingly. As a result, the instantaneous amplitude fluctuations of the pulses are suppressed. Thus compressing the pulse width and equalizing the pulse train amplitudes can be realized simultaneously.

\subsection{Numerical Simulation}

Evolution of pulse width as it makes the round trips in the cavity can be simulated by solving the generalized nonlinear Schrödinger equation (GNLSE) using split-step Fourier method [48-50]. Figure 14 shows the evolution of pulse width (as it makes the round trips in the cavity) with different polarization configurations (corresponding to different $\alpha 2$ values). Figure 14(a) is the active mode-locking only case and the generated pulse width is $\sim 5.63 \mathrm{ps}$. In Figure 14(b) the NPR works strongly as a pulse compressor and the generated pulse width 
is compressed to $\sim 1.88$ ps. In Figure 14(c) we can see, after some cycles, the pulse width is broadened to $\sim 6.56 \mathrm{ps}$. The NPR in this state only acts as an amplitude equalizer and as a result the pulse width is broadened. In Figure 14(d), the NPR does not affect the pulse width significantly and the output pulse width is $\sim 5.25 \mathrm{ps}$.

(a)

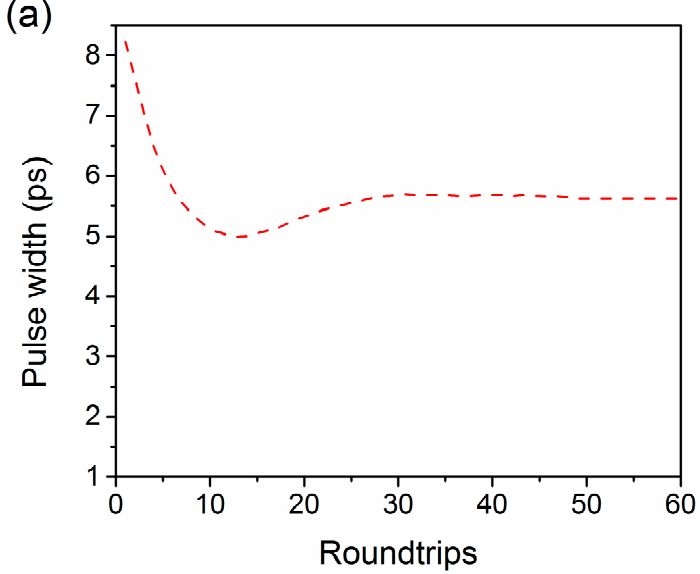

(c)

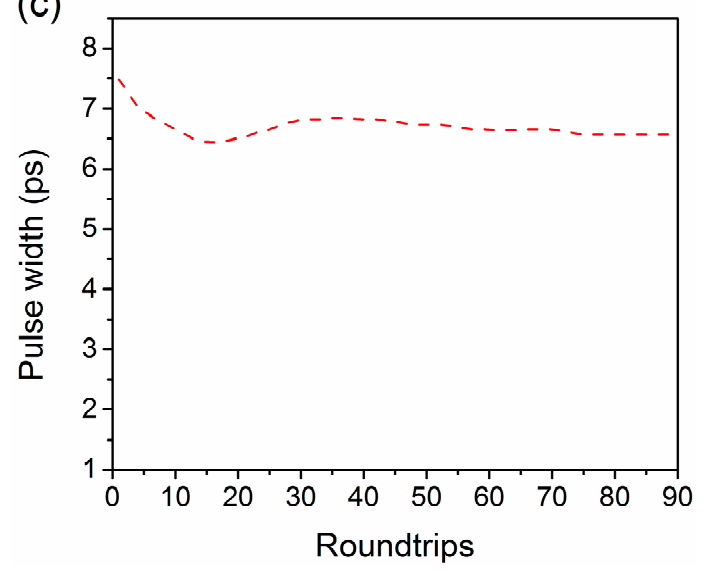

(b)

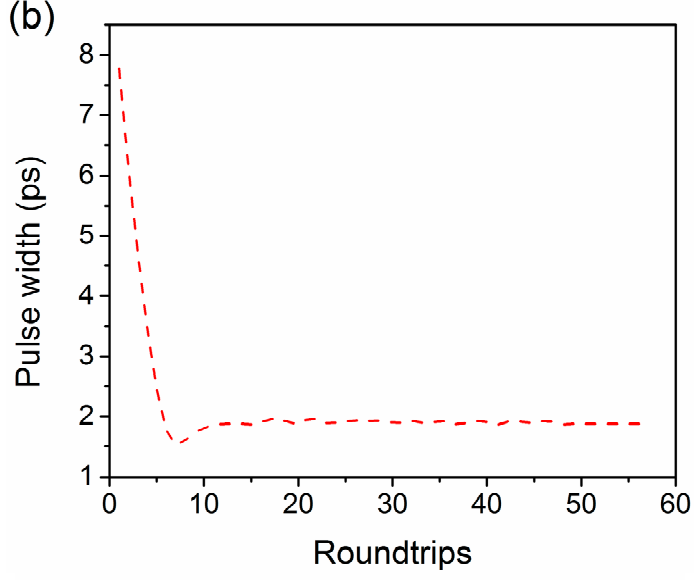

(d)

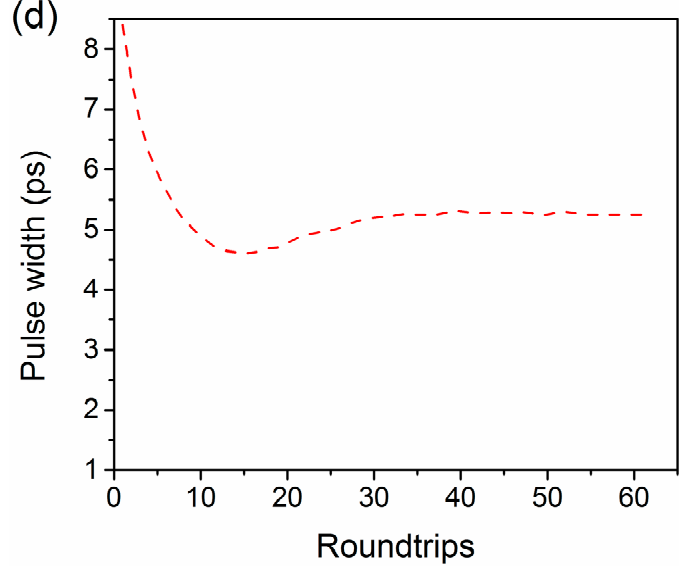

Figure 14. Numerical simulation results of the evolution of the pulse width in the fiber ring laser. (a) Only rational active harmonic mode-locking. (b) Hybrid mode-locking with NPR, $\alpha_{2}=30^{\circ}$. (c) $\alpha_{2}=120^{\circ}$. (d) $\alpha_{2}=85^{\circ}$.

\subsection{Fiber Laser Experimental result}

After carefully tuning the PCs and the frequency of the RF signal, a $30 \mathrm{GHz}$ pulse train with ultrashort pulse width can be generated. If we remove polarization controllers PC1, PC2 and the polarizer, we can assume that the NPR has no effect on pulse shaping or there is no NPR in the cavity. Figure 15 shows the auto-correlation trace of the output pulse train with only RHML (a) and of hybrid mode-locking with NPR (b). Without NPR, the generated pulse train has a calibrated pulse width $\sim 5.8$ ps which is very close to the numerical calculated value $5.63 \mathrm{ps}$. However, with NPR in cavity and after the careful tuning of the PCs, the pulse width of the generated $30 \mathrm{GHz}$ pulse train is shortened to $\sim 1.9 \mathrm{ps}$. The compressing ratio is as high as $67 \%$. The NPR inside the cavity can greatly improve the pulse shortening mechanism of the fiber ring laser due to the high loss it induced to the pulse wings. The central part of the pulse experiences relatively low loss compared to the pulse wings. This agrees well with the numerical simulation as it shows that with $\alpha_{2}=30^{\circ}$, the pulse width could be compressed to $1.88 \mathrm{ps}$. 

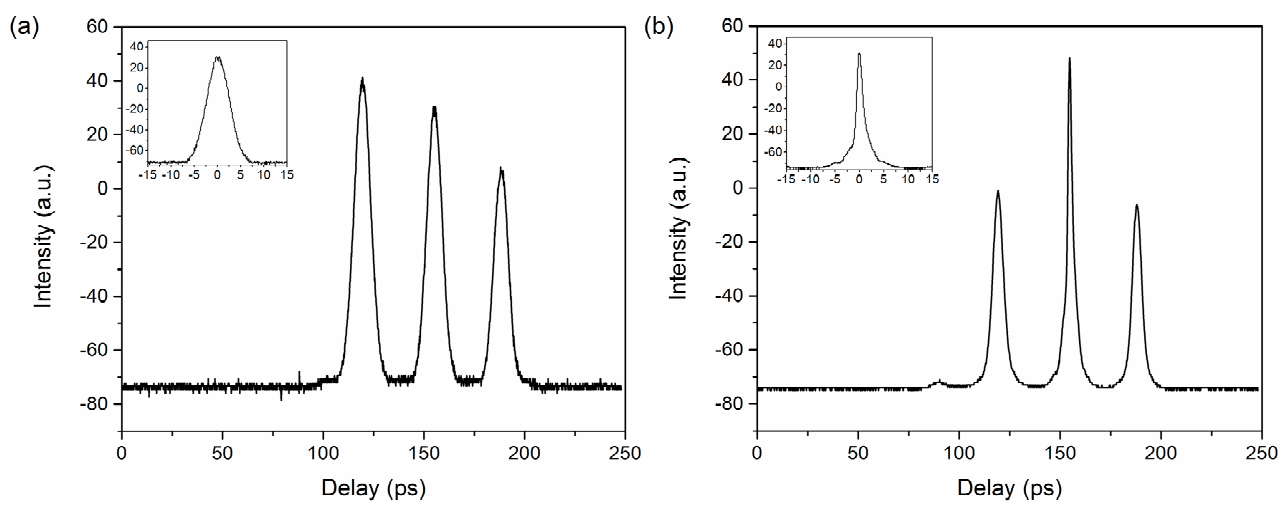

Figure 15. Auto-correlation trace of the pulse train output from the fiber ring laser. (a) With only rational harmonic mode-locking, the $30 \mathrm{GHz}$ pulse width is $\sim 5.8$ ps. (b) Hybrid mode-locking with NPR in the cavity, the $30 \mathrm{GHz}$ pulse width is compressed to $\sim 1.9 \mathrm{ps}$.

Figure 16 compares the RF spectra of the generated $30 \mathrm{GHz}$ pulse train with only RHML (a) and of hybrid mode-locking with NPR (b). The highest peak in both figures correspond to the operating frequency of the fiber ring laser which is $\sim 30 \mathrm{GHz}$. As we can see in Figure 16(a), without NPR, there are sidebands in the RF spectrum. These are the supermodes which cannot be removed by tuning the modulation frequency $\mathrm{fm}_{\mathrm{m}}$ and the polarization controller PC3 in the cavity. However, no supermodes are observed in Figure 16(b) with the presence of NPR in the cavity. The signal-to-noise ratio is over $25 \mathrm{~dB}$ after the NPR incorporated. Because the supermodes competition directly leads to pulse fluctuation between adjacent peaks, the reduction of the supermodes in the RF spectrum can improve the stability of optical pulse generation in high order rational harmonic mode locking [51].
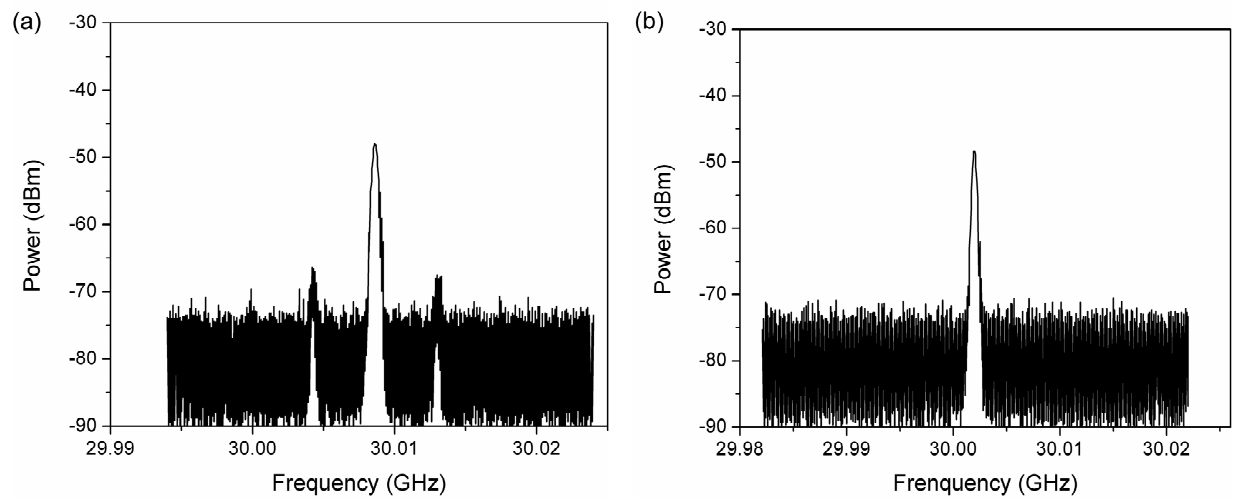

Figure 16. RF spectra of the generated pulse trains at $30 \mathrm{GHz}$. (a) only rational harmonic mode-locking. (b) Hybrid mode-locking with NPR in the cavity.

\section{Summary and Perspective}

Hybrid mode-locked fiber ring lasers which combine rational harmonic mode locking technique and saturable absorber inside ring cavity have been designed and experimentally demonstrated by using different schemes such as charcoal nano-particles, graphene and nonlinear polarization rotation in photonic crystal fiber. These hybrid mode-locked lasers are able to generate optical pulse train with improved stability (less supermodes), shortened pulse width ( $\sim 1 \mathrm{ps})$ and high repetition rate (20 to $40 \mathrm{GHz})$. Numerical method to simulate the pulse evolution inside ring cavity has also been proposed by solving nonlinear Schrödinger 
equations. The hybrid mode locking technique is a very promising way to generate ultrashort optical pulse train with high date rate. Further investigation on hybrid mode locking schemes to generate optical pulse train with pules width less than $1 \mathrm{ps}$ and repetition rate higher than $100 \mathrm{GHz}$ are required for future optical communication system.

\section{Conflict of Interest}

The authors declare no conflict of interest.

\section{References}

1. W. Li, "High Speed All Optical Switching and Encryption using Ultrafast Devices" (2015). Doctoral Dissertations. 956. http://digitalcommons.uconn.edu/dissertations/956

2. C. Wu and N. K. Dutta, "High-repetition-rate optical pulse generation using a rational harmonic mode-locked fiber laser," Quantum Electronics, IEEE Journal of 36, 145-150 (2000).

3. M. N. Vinoj and V. C. Kuriakose, "Generation of pedestal-free ultrashort soliton pulses and optimum dispersion profile in real dispersion-decreasing fibre”, J. of Opt. A: Pure and Appl. Optics, 6, 1, 63-70 (2004).

4. G. R. Lin, Y. C. Lin, K. C. Lin, W. Y. Lee and C. L. Wu, "50 fs soliton compression of optical clock pulse recovered from NRZ data injected SOAFL", Opt. Express, 18, 9, 9525-9530 (2010).

5. K. Taira and K. Kikuchi, "Subpicosecond pulse generation using an electroabsorption modulator and double-stage pulse compressor", IEEE Photon. Technol. Lett., 15, 9, 12881290 (2003).

6. K. Taira and K. Kikuchi, "Picosecond pulse generation with high extinction ratio employing electroabsorption modulator, fiber compressor and self-phase modulationbased reshaper," Electron. Lett., 40, 1, 15-16 (2004).

7. Y. Ozeki, S. Takasaka, T. Inoue, K. Igarashi, J. Hiroishi, R. Sugizaki, M. Sakano and S. Namiki, "Nearly exact optical beat-to-soliton train conversion based on comb-like profiled fiber emulating a polynomial dispersion decreasing profile," IEEE Photon. Technol. Lett., 17, 8, 1698-1700 (2005).

8. Y. Ozeki, S. Takasaka, J. Hiroishi, R. Sugizaki, T. Yagi,M. Sakano and S. Namiki, "Generation of $1 \mathrm{THz}$ repetition rate, $97 \mathrm{fs}$ optical pulse train based on comb-like profiled fibre," Electron. Lett., 41, 19, 1048-1050 (2005).

9. C. De Dios and H. Lamela, "Compression and Reshaping of Gain-Switching LowQuality Pulses Using a Highly Nonlinear Optical Loop Mirror,” IEEE Photon. Technol. Lett., 22, 6, 377-379 (2010).

10. S. Ma, W. Li, H. Hu, and N. K. Dutta, "High speed ultrashort-pulse fiber ring laser using photonic crystal fiber nonlinear optical loop mirror," Opt. Commun., 285 (12), 2832-2835 (2012)

11. S. Ma, W. Li, H. Hu, and N. K. Dutta, "Ultra-short pulsed fiber ring laser using photonic crystal fiber", SPIE Defense, Security, and Sensing, 838116-838116-4 (2012)

12. O. Okhotnikov, A. Grudinin, and M. Pessa, "Ultra-fast fibre laser systems based on SESAM technology: new horizons and applications," New journal of physics 6, 177 (2004).

13. 10. X. He, Z.-b. Liu, and D. Wang, "Wavelength-tunable, passively mode-locked fiber 
laser based on graphene and chirped fiber Bragg grating," Optics letters 37, 2394-2396 (2012).

14. 11. J. L. Luo, L. Li, Y. Q. Ge, X. X. Jin, D. Y. Tang, S. M. Zhang, and L. M. Zhao, "LBand Femtosecond Fiber Laser Mode Locked by Nonlinear Polarization Rotation," Photonics Technology Letters, IEEE 26, 2438-2441 (2014).

15. 12. X. Liu, T. Wang, C. Shu, L. Wang, A. Lin, K. Lu, T. Zhang, and W. Zhao, "Passively harmonic mode-locked erbium-doped fiber soliton laser with a nonlinear polarization rotation," Laser physics 18, 1357-1361 (2008).

16. 13. A. F. Runge, C. Aguergaray, R. Provo, M. Erkintalo, and N. G. Broderick, "Allnormal dispersion fiber lasers mode-locked with a nonlinear amplifying loop mirror," Optical Fiber Technology 20, 657-665 (2014).

17. W. Li, H. Hu, X. Zhang, S. Zhao, K. Fu, and N. K. Dutta, "High-speed ultrashort pulse fiber ring laser using charcoal nanoparticles”, Applied Optics, 55 (9), 2149-2154 (2016)

18. X. Zhang, H. Hu, W. Li, and N. K. Dutta, "High-repetition-rate ultrashort pulsed fiber ring laser using hybrid mode locking”, Applied Optics, 55(28), 7885-7891 (2016)

19. H. Hu, X. Zhang, W. Li, and N.K. Dutta, "Hybrid mode-locked fiber ring laser using graphene and charcoal nanoparticles as saturable absorbers", SPIE Defense+ Security, 983630-983630-8 (2016)

20. S. Yamashita, "A tutorial on nonlinear photonic applications of carbon nanotube and graphene,” J. Lightwave Technol. 30(4), 427-447 (2012).

21. B.V. Cunning, C. L. Brown, and D. Kielpinski, "Low-loss flake-graphene saturable absorber mirror for laser mode-locking at sub-200-fs pulse duration," Appl. Phys. Lett. 99(26), 261109(2011).

22. G. Sobon, J. Sotor, and K. M. Abramski, “All-polarization maintaining femtosecond Erdoped fiber laser mode-locked by graphene saturable absorber," Laser Phys. Lett. 9(8), 581-586(2012).

23. D. Popa, Z. Sun, F. Torrisi, T. Hasan, F. Wang, and A. C. Ferrara, "Sub 200fs pulse generation from a graphene mode-locked fiber laser,” Appl. Phys. Lett. 97(20), 203106 (2010).

24. J. Xu, S. Wu, H. Li, J. Liu, R. Sun, F. Tan, H. Yang, and P. Wang, "Dissipative solition generation from a graphene oxide mode-locked Er-doped fiber laser," Opt. Express. 20(21), 23653-23658(2012).

25. Z.B. Liu, X. He, and D. N. Wang, "Passively mode-locked fiber laser based on a hollowcore photonic crystal fiber filled with few-layered graphene oxide solution," Opt. Lett. 36(16), 3024-3026(2011).

26. Y. H. Lin and G.-R. Lin, "Kelly sideband variation and self four-wave-mixing in femtosecond fiber soliton laser mode-locked by multiple exfoliated graphite nanoparticles," Laser Phys. Lett. 10(4), 045109 (2013).

27. V. V. Singh, G. Gupta, A. Batra, A. K. Nigam, M. Boopathi, P. K. Gutch, B. K. Tripathi, A. Srivastava, M. Samuel, G. S. Agarwal, B. Singh, and R. Vijayaraghava, "Greener electrochemical synthesis of high quality graphene nanosheets directly from pencil and its SPR sensing application," Adv. Funct. Mater. 22(11), 2352-2362(2012).

28. Y. H. Lin, Y. C. Chi, and G.-R. Lin, "Nanoscale charcoal powder induced saturable absorption and mode-locking of a low-gain erbium-doped fiber-ring laser," Laser Phys. Lett. 10(5), 055105 (2013)

29. Y. H. Lin, Y. C. Chi, and G-R. Lin, "Nanoscale charcoal powder induced saturable 
absorption and mode-locking of a low-gain erbium-doped fiber-ring laser," Laser Phys. Lett. 10(5), 055105(2013)

30. H. Hu, W. Li, S. Ma, and N. K. Dutta, "Coherence Properties of Supercontinuum Generated in Dispersion-Tailored Lead-Silicate Microstructured Fiber Taper," Fiber and Integrated Optics, 32 (3), 209-221 (2013)

31. H. Hu, W. Li, and N. K. Dutta, "Wide-band Coherent Supercontinuum generation," SPIE Defense, Security, and Sensing, 87330C-87330C-8 (2013)

32. H. Hu, W. Li, and N. K. Dutta, "Supercontinuum generation in dispersion-managed tapered-rib waveguide," Applied Optics, 52 (30), 7336-7341 (2013)

33. H. Hu, W. Li, and N. K. Dutta, "Supercontinuum Generation in a Tapered Rib Waveguide," SPIE Optical Engineering and Application, 92021C-92021C-10 (2014)

34. Set, S.Y., Yaguchi, Hiroshi, Tanaka, Y., Jablonski, M., "Laser mode locking using a saturable absorber incorporating carbon nanotubes," Journal of Lightwave Technology 22(1), 51-56 (2004).

35. Jaroslaw Sotor, Grzegorz Sobon, Wojciech Macherzynski, Piotr Paletko, Kacper Grodecki, and Krzysztof M. Abramski, "Mode-locking in Er-doped fiber laser based on mechanically exfoliated $\mathrm{Sb}_{2} \mathrm{Te}_{3}$ saturable absorber," Opt. Mater. Express 4(1), 1-6 (2014).

36. Handing Xia, Heping Li, Changyong Lan, Chun Li, Xiaoxia Zhang, Shangjian Zhang, and Yong Liu, "Ultrafast erbium-doped fiber laser mode-locked by a CVD-grown molybdenum disulfide $\left(\mathrm{MoS}_{2}\right)$ saturable absorber," Opt. Express 22(14), 17341-17348 (2014).

37. Bao, Q., Zhang, H., Wang, Y., Ni, Z., Yan, Y., Shen, Z. X., Loh, K. P. and Tang, D. Y., "Atomic-Layer Graphene as a Saturable Absorber for Ultrafast Pulsed Lasers," $A d v$. Funct. Mater., 19, 3077-3083 (2009).

38. Chang, Y. M., Kim, H., Lee, J. H., and Song, Y. W., "Multilayered graphene efficiently formed by mechanical exfoliation for nonlinear saturable absorbers in fiber mode-locked lasers," Applied Physics Letters 97(21), 211102 (2010).

39. Sobon, Grzegorz, Jaroslaw Sotor, and Krzysztof M. Abramski, "Passive harmonic modelocking in Er-doped fiber laser based on graphene saturable absorber with repetition rates scalable to 2.22 GHz," Applied Physics Letters 100(16), 161109 (2012).

40. Martinez, Amos, and Shinji Yamashita, "10 GHz fundamental mode fiber laser using a graphene saturable absorber," Applied Physics Letters 101(4), 041118 (2012).

41. H. Hu, W. Li, and N. K. Dutta, "Dispersion-engineered tapered planer waveguide for coherent supercontinnuum generation," Opt Commun.,324,252-257, (2014)

42. H. Hu, W. Li, X. Zhang, and N. K. Dutta, "Simulation of octave spanning mid-infrared supercontinuum generation in dispersion-varying planar waveguides", Applied Optics, 54(11), 3448-3454 (2015)

43. H. Hu, W. Li, X Zhang, and N. K. Dutta, "Broadband Supercontinuum Generation in a Tapered-Rib Lead-Silicate Waveguide", International Journal of High Speed Electronics and Systems, 24, 1550009 (2015)

44. J. L. Luo, L. Li, Y. Q. Ge, X. X. Jin, D. Y. Tang, S. M. Zhang, and L. M. Zhao, "L-Band Femtosecond Fiber Laser Mode Locked by Nonlinear Polarization Rotation," Photonics Technology Letters, IEEE 26, 2438-2441 (2014).

45. X. Liu, T. Wang, C. Shu, L. Wang, A. Lin, K. Lu, T. Zhang, and W. Zhao, "Passively harmonic mode-locked erbium-doped fiber soliton laser with a nonlinear polarization rotation," Laser physics 18, 1357-1361 (2008) 
46. F. Adler, K. Moutzouris, A. Leitenstorfer, H. Schnatz, B. Lipphardt, G. Grosche, and F. Tauser, "Phase-locked two-branch erbium-doped fiber laser system for long-term precision measurements of optical frequencies," Optics express 12, 5872-5880 (2004).

47. Z. Li, C. Lou, K. T. Chan, Y. Li, and Y. Gao, "Theoretical and experimental study of pulse-amplitude-equalization in a rational harmonic mode-locked fiber ring laser," IEEE Journal of Quantum Electronics 37, 33-37 (2001).

48. X. Zhang, H. Hu, W. Li, and N. K. Dutta, "Mid-infrared supercontinuum generation in tapered As2S3 chalcogenide planar waveguide", Journal of Modern of Optics, 63 (19), 1965-1971(2016)

49. X. Zhang, H. Hu, W. Li, and N. K. Dutta, "Broadband on-chip mid-IR supercontinuum generation", SPIE Defense+ Security, 983211-983211-8 (2016)

50. X. Zhang, H. Hu, W. Li, and N. K. Dutta, "Supercontinuum generation in dispersionvarying microstructured optical fibers", SPIE Defense+ Security, 98340F-98340F-7 (2016)

51. C. Wu and N. K. Dutta, "High-repetition-rate optical pulse generation using a rational harmonic mode-locked fiber laser," Quantum Electronics, IEEE Journal of 36, 145-150 (2000). 